\title{
Discursos de género en Contact Improvisation Deliberación y acción coreográfica en torno a la revista Contact Quarterly*
}

Recibido: 27/08/2019 | Revisado: 03/12/2019 | Aceptado: 17/03/2020

DOI: $10.17230 /$ co-herencia.17.33.5

\section{María Paz Brozas-Polo** \\ mpbrop@unileon.es}

Resumen La técnica de danza colectiva Contact Improvisation surgió hace casi medio siglo en Nueva York como una alteración de los códigos de contacto y de las jerarquías coreográficas; desde las primeras décadas de su desarrollo -ideológico, pedagógico o estético-, se viene produciendo una compleja discusión en torno al género. En este trabajo se analizan las distintas perspectivas del debate que se articulan en lo que se puede considerar la revista fundacional, Contact Quarterly: el cuestionamiento de la masculinidad, las ideas de neutralización y de minimización de lo sexual y varios enfoques feministas entre los que se puede incluir la teoría queer. Más que en la definición de cada una de las posiciones, nos detenemos en la observación crítica de las paradojas, tensiones y puntos de encuentro que se van reflejando en la práctica y van tejiendo una fecunda red discursiva.

\section{Palabras clave:}

Contacto, improvisación, danza, cuerpo, debate, género, historia, revista Contact Quarterly.

\section{Gender Discourses in Contact Improvisation Debate and Choreographic Action in the Contact Quarterly}

\footnotetext{
Abstract The technique of group dance named Contact Improvisation emerged almost half a century ago in New York as an alteration of contact codes and choreographic hierarchies. From the first decades of its development (i.e., ideological, pedagogical, or aesthetic), there has been a complex debate regarding gender. In this study, the different perspectives in the debate are analyzed, which are articulated in - what can be deemed as a foundational journalContact Quarterly: questioning of masculinity, the ideas of
}

Esta investigación fue realizada entre 2018 y 2020 con el apoyo de la Universidad de León: Departamento de Educación Física y Deportiva, Biblioteca y Aula de Artes del Cuerpo -Área de Actividades Culturales-.

** Profesora Titular de la Universidad de León, España. ORCID: 0000-0003-0117-3117 
neutralization and minimalization of sexual topics, and various feminist approaches, as well as queer theory. Rather than the definitions of each one of the postures, we focus on the critical observation of paradoxes, tensions, and points of contact that are reflected in the practice and knit a prolific discourse net.

\section{Keywords:}

Contact, improvisation, dance, body, debate, gender, history, Contact Quarterly journal.

\section{El discurso coreográfico y la improvisación colectiva}

Los discursos habitan en los cuerpos: las prácticas corporales y, cómo no, las coreográficas se alimentan de discursos y a su vez son generadoras de discurso. En este sentido, la construcción coreográfica se puede definir como una construcción de discurso y la propia danza, en tanto que interpretación de una coreografía, se puede entender, a su vez, como lectura o matización del discurso creado por el coreógrafo. Si atendemos en particular a los discursos de género, las elecciones de quien se dedica a coreografiar ponen de manifiesto su teorización sobre el cuerpo; es decir, al coreografiar se define un repertorio de acciones corporales que puede confirmar o contravenir las expectativas sociales de género. De forma particular, Foster (1998) se refiere explícitamente a los códigos de contacto, postura y movimiento que se establecen en la coreografía colectiva, donde se elabora no solo una teoría de la identidad corporal de género, sino una propuesta de las relaciones de género entre los cuerpos; así lo explica en alusión a la labor discursiva de una supuesta coreógrafa que experimenta en su estudio:

She reckons with established codes of contact between female and male bodies: where the body of one sex can touch the body of the other sex, what kinds of shapes bodies the two sexes can make together, who can give weight and who bear it, who initiates movement and who follows, who is passive and who active, who is to be looked at and who is doing the looking. She forges phrases of movement that construct groupings of dancers with gendered connotations -chaotic, convoluted, pristine, or geometric. When she does this for multiple bodies, she elaborates a theory not only of gendered corporeal identity but also of relations among gendered bodies (Foster, 1998, p. 8). 
En el caso del Contact Improvisation (CI), la forma coreográfica experimental que nos ocupa en este artículo, la exploración se produce a través de una articulación de los cuerpos muy definida: se trata de un movimiento improvisado derivado del diálogo físico por medio del contacto directo y, más precisamente, del diálogo entre los pesos. En una de sus primeras publicaciones, Paxton define el Contact Improvisation como un sistema dual en el que "cada parte del dúo improvisa libremente tratando de usar los caminos disponibles más fáciles para que sus masas se muevan mutuamente" (1975, p. 40; mi traducción).

La reivindicación del Contact Improvisation en su origen -años 70 en Nueva York (Novack, 1990)- afectó a la concepción misma de la danza en la medida en que parecían invertirse o al menos cuestionarse los códigos opuestos clásicos como mente/cuerpo, masculino/femenino, estructura/improvisación, coreografía/proceso, pensamiento/acción, etcétera, sobre los que nuestra tradición había acentuado los valores de la izquierda (Rizzo, 1998; Brozas, 2000).

Además de desestabilizar las jerarquías coreográficas binarias, esta práctica introdujo la alteración de las distancias sociales tal como fueron definidas por Hall en el contexto estadounidense de mediados del siglo XX en su teoría sobre la proxemia. A este respecto, Novack (1990) se refiere a los usos del espacio en Contact Improvisation equiparándolos a los de las relaciones íntimas propias de las relaciones sexuales, del cuidado de enfermos o de la crianza de bebés. Karen Nelson (2008 [1996], p. 105) lo explica, asimismo, mediante lo que denomina "la revolución del tacto" que lleva consigo esta forma de improvisación. En este sentido, Nelson (2008, p. 103) propuso definir el Contact Improvisation como política del movimiento puesto que organiza la forma de romper el código de las distancias entre los cuerpos: abrir la posibilidad de compartir el movimiento en un contexto no sexual significaría cambiar el rostro de la convivencia social. Ciertamente, en esta técnica de contacto en movimiento, a diferencia de otras, no existen roles de género y no hay una asignación específica sobre quién toca y quién es tocado, quien guía y quién es guiado y quién sostiene el cuerpo del otro. Se interpela a los cuerpos de los participantes como dispositivos disponibles a los que se les presume con una potencialidad enorme, a los que se reclama un estado de suma atención y una movilidad 
multidireccional no discriminatoria. Aun siendo, como se indica, bien preciso el foco de este juego de movimiento -la exploración del desplazamiento del peso compartido a través del contacto- se trata de una forma de danza muy abierta que permite toda una matización e incluso reconstrucción coreográfica; pensamos que es justamente esta apertura la que posibilita cierta generación, degeneración o regeneración del discurso inicial en cada cuerpo o grupo que baila y que organiza los dispositivos pedagógicos o performativos que contextualizan el baile.

Siguiendo una pauta cronológica, y apoyándonos en el eje vertebrador que constituye la revista Contact Quarterly -A vehicle for moving ideas. Journal of Dance and Improvisation-, esta investigación pretende abordar aquellos discursos de género que se pueden identificar y relacionar desde el comienzo de la práctica del Contact Improvisation en los años 70 hasta la actualidad. Nos preguntamos, cuáles son las teorías que atraviesan la experiencia del Contact Improvisation y cómo se articulan con la acción colectiva; además de identificar y comprender las distintas perspectivas de género, sexualidad o identidad, nuestra intención es subrayar el interés -no solo epistemológico- de la existencia de un debate abierto en el que se manifiestan afinidades y discrepancias; este artículo es, en cierta medida, una reivindicación de dicho debate en sus distintas expresiones.

Como practicante y enseñante de Contact Improvisation y, asimismo, como investigadora, no dejo de admirar el compromiso de sucesivas generaciones que han desarrollado ingente conocimiento y construido, colectivamente, espacios de encuentro para el diálogo; entre ellos, la revista Contact Quarterly puede considerarse un espacio fundacional.

\section{Contact Quarterly: discusión a través de un archivo}

A medida que la propuesta coreográfica derivada del Contact Improvisation se ha ido extendiendo y reconfigurando en distintos contextos, han ido proliferando interrogantes sobre su propia definición, así como sobre otras cuestiones políticas y pedagógicas entre las que se encuentran las relacionadas con el género y con la 
sexualidad. Gran parte de las inquietudes asociadas a la expansión de la práctica se han publicado en la revista Contact Quarterly (CQ). Esta revista dedicada a la danza y a la improvisación, que se puso en marcha en 1975, es un archivo fundamental que materializa y contiene buena parte del debate en el que tratamos de sumergirnos. El nombre inicial de la revista fue Contact Newsletter (1975-76), pasando un año después a denominarse Contact Quarterly. Concebida como un noticiero sobre talleres y performances de Contact Improvisation se convirtió en una publicación bianual miscelánea, quedando las Newsletter como una sección informativa. La editorial de la revista -Contact Editions- ha publicado, además, tres volúmenes recopilatorios -Contact Improvisation Sourcebook- compuestos exclusivamente por artículos de Contact Improvisation -con las secciones Still Moving y Essentials-, y que excluyen la sección Newsletter así como los textos sobre otras temáticas de danza, improvisación o técnicas somáticas también incluidas en la sección Out in the Field ${ }^{1}$ para este estudio hemos utilizado los dos volúmenes que contienen las publicaciones relativas a 1975-1992 y 1993-2007 y se han consultado uno a uno el resto de los números hasta la actualidad gracias a la suscripción de la Universidad de León.

El objetivo inicial de la revista fue facilitar el intercambio colectivo y el mutuo enriquecimiento, así como reflexionar sobre la definición y la pedagogía, es decir, crear un foro para debatir los sentidos y los procesos de la propia práctica:

From a single week of performance in 1972, CI has blossomed into a movement form practiced by thousands around the world. CQ has tried to represent the broad diversity of voices that benefit from and contribute to the form in hopes of not only documenting but also encouraging exchange within the field. We hope this collection continues to inform and inspire your practice of Contact Improvisation (Stark Smith, 2008, p. IX).

Otras funciones de la revista fueron cuidar a los practicantes de las lesiones, atendiendo a ciertos riesgos físicos derivados del entrenamiento, así como apoyar a los docentes sin restringir el acceso a la enseñanza ni ejercer un dominio por medio de un copyright -un asunto delicado y controvertido-:

1 (https://contactquarterly.com/) 
In 1975, three years into the practice of Contact Improvisation, we began hearing of physical injuries resulting from people doing Contact from having seen it in performance without an introduction to the subtle sensing work that underlies the more vigorous physical activity. How to keep the work safe? we wondered. Steve Paxton, and the small group continuing to practice and show the work with him, considered trademarking the name and certifying or authorizing teachers. Preliminary papers were drawn up but were never signed. The prospect of policing Contact was not appealing. More interesting was the idea of creating a vehicle for communication in which to report activity and current thinking within the work, to keep the work open by inviting ourselves and others further into the dialogue. Thus, the Contact Newsletter began. (Later to become Contact Quarterly). This decision not to trademark the name, and instead to encourage communication, seems to have been a crucial one, leading to the considerable expansion and enrichment of the work by many individuals over the years (Stark Smith, 2008 [1998], p. 177).

Si revisamos longitudinalmente los artículos que tratan el tema del género, se puede observar una presencia tímida y menos enfocada en las primeras décadas, una atención específica en dos monográficos de 1996 dedicados al tema "sexualidad e identidad", y una eclosión particular en los últimos años -sin discontinuidad desde 2017coincidiendo con la expansión del debate en otros foros. A efectos expositivos, hemos sintetizado esta evolución en tres etapas (véase tabla 1). Algunos interlocutores se mantienen activos a lo largo de los años: las propias editoras y en particular Nancy Stark Smith o el propio Steve Paxton -considerado principal autor del Contact Improvisation- dialogan con otros bailarines como, por ejemplo, Keith Hennessy, quien mantiene su atención permanente en las políticas de género y sexualidad. No obstante, también se detecta la incorporación constante de nuevas voces que van enriqueciendo las posturas y argumentaciones; del mismo modo, la renovación de los comités editoriales también se refleja en los contenidos de la misma. El año 2008 se puede considerar un hito debido a la 
aparición de la sección Unbound CQ, que es una ampliación online para subscriptores (M. Bove, comunicación personal, julio 1, 2019). Recientemente se ha editado y publicado el último número en papel -2020 (45, 1)- para darle paso así a una versión exclusivamente digital de la revista. En cuanto a los modos de producción de artículos, se organizan en función de las distintas secciones en las que se estructura la revista e incluyen variados formatos, tamaños y fuentes, siendo frecuentes las firmas colectivas y ocasionales las anónimas: pocos textos se asimilan al ensayo académico, predominan los relatos de experiencias personales o retroacciones de equipos de trabajo tras encuentros, laboratorios, festivales u otras actividades colectivas; en ocasiones se prolongan los debates en números sucesivos con reformulaciones y réplicas.

\section{Tabla 1.}

Etapas del debate de género en Contact Quarterly 1975-2019

\begin{tabular}{|c|c|}
\hline Contact Quarterly & Año, vol.(n. $\left.{ }^{\circ}\right)$, autores, título \\
\hline \multirow{10}{*}{$\begin{array}{l}\text { Political and Sexual } \\
\text { Issues } \\
1975-1995\end{array}$} & 1978, 3(3-4), Brown, "The Politics of Contact" \\
\hline & 1987, 12(3), Horwitz, "The Politics of Improvisation" \\
\hline & 1988, 13(3), Ormand, “The Berlin Jam” \\
\hline & $\begin{array}{l}\text { 1988, 13(3), Novack, "Egalitarianism and hierarchy in } \\
\text { Contact Improvisation" }\end{array}$ \\
\hline & 1989, 14(1), Paxton, "Hier-Visibility" \\
\hline & 1990, 15(2), Elliot, "Making Safe Contact" \\
\hline & $\begin{array}{l}\text { 1990, 15(1), Keogh \& Andrews (eds.), "What's in the bag?" } \\
\text { Transcript of discussion of issues in the shadow of Contact }\end{array}$ \\
\hline & 1994, 19(1), Nelson, “Dear CQ. Arena for discussion” \\
\hline & 1994, 19(2), Lori B, “Sex Talk at Harbin” \\
\hline & 1995, 20(2), Koening \& Mattson, "Hierarchy and Elitism" \\
\hline
\end{tabular}




\begin{tabular}{|c|c|}
\hline \multirow{19}{*}{$\begin{array}{l}\text { Focus on Sexuality } \\
\& \text { Identity } \\
\text { 1996: 21(1), 21(2) }\end{array}$} & 1996, 21(1), Fulkerson, "Taking the Globe Without the Hand" \\
\hline & $\begin{array}{l}\text { 1996, 21(1), Lori B \& Paxton, "The Sex Issue: Dialogue } \\
\text { about Sex \& Contact Improvisation" }\end{array}$ \\
\hline & 1996, 21(1), Curtis, "Why Talk (Dance, Paint) About Sex?" \\
\hline & 1996, 21(1), Anonymous, "Speaking of Boundaries..." \\
\hline & 1996, 21(1), Truss, "What Kind of Touch is This?" \\
\hline & 1996, 21(1), Cashman, "Personal Boundaries in Contact" \\
\hline & 1996, 21(1), Nelson, "Touch Revolution: Giving Dance" \\
\hline & 1996, 21(1), Hennessy, "Love and Sex, Touch and Weight" \\
\hline & 1996, 21(1), Zientara, "Sweatpants Grope My Memory" \\
\hline & $\begin{array}{l}\text { 1996, 21(2), Stark Smith, "Riding the Wild Beast Back to } \\
\text { Its Den" }\end{array}$ \\
\hline & $\begin{array}{l}\text { 1996, 21(2), Langland, "Between the Cracks: Interview with } \\
\text { Diane Torr on Gender \& Sexual Identity [...]" }\end{array}$ \\
\hline & $\begin{array}{l}\text { 1996, 21(2), Schmitt, "Contexting Contact and Other } \\
\text { Non-answers" }\end{array}$ \\
\hline & 1996, 21(2), Waters, "Back to Physics" \\
\hline & $\begin{array}{l}\text { 1996, 21(2), McAlpin \& Vashon, "Dance, Intimacy, Touch, } \\
\text { and Pleasure" }\end{array}$ \\
\hline & 1996, 21(2), Paxton, “...To Touch” \\
\hline & $\begin{array}{l}\text { 1996, 21(2), Heitkamp, "School of Sensitivity: Contact \& } \\
\text { Sexuality Workgroup" }\end{array}$ \\
\hline & $\begin{array}{l}\text { 1996, 21(2), Rounds, "Dancing with the Moon: Contact } \\
\text { Improvisation within a Female Body" }\end{array}$ \\
\hline & $\begin{array}{l}\text { 1996, 21(2), Nirino \& Olender, "Buenos Aires, Argentina: } \\
\text { Land of Dance and Warm People" }\end{array}$ \\
\hline & $\begin{array}{l}\text { 1996, 21(2), Adkins \& Mang, "The Social Context of Con- } \\
\text { tact Improvisation" [Report on a Study Lab] }\end{array}$ \\
\hline
\end{tabular}


1999, 24(1), Frost, "Some Short Stories about Sex and Contact"

2003, 28(2), Prince, "Leave Your Identity at the Door?: Politics of Difference and Contact Improvisation"

2003, 28(2), Keogh, "101 Ways to Say No to Contact Improvisation: Boundaries and Trust"

2004, 29(1), Anonymous, "Reflections on Midlife: Contact Improvisation and Falling in Love"

2008, (Unbound), Hechler, "Heteronormativity in Contact Improvisation"

2009, 34(1), Zolbrod, "In Response to Robert Turner's 'Freedom? Power? Happiness?: Neuro-Politics of Cl': Notes in a Post-Talk Discussion at CI36"

2009, 34(2), Boulder Lab Core, "Deepening through Contact Improvisation: The Boulder Contact Lab Guidelines" 2012, 37(1), Hennessy, "Post/Contact: Seven class descriptions proposing ongoing experiments in dancing and performing under the influence of Contact Improvisation"

Twenty-first century and CQ 2013, 38(1), Mang, Mägi, Hansen, \& Gartner, "Radical Unbound from Contact: A Meeting on Contact Improvisation and Body Politics"

2008 2014, 39(1), Rythea, "Contact Improvisation: One Experience" 2014, 39(1), Henessy, "848 Community Space: Queer, Sex, Performance, and contact improvisation in 1990s San Francisco"

2014, 39(2), CQ Eds, (CQ Contact Improvisation Newsletter), "Jam Guidelines"

2017, 42(1), Horrigan, "Queering Contact Improvisation"

2017, 42(2), Yardley, "Respecting Boundaries/Coexisting Genders: Zine about Women's Experiences of Feeling Unsafe in Contact Improv"

2018, 43(1), Stark Smith \& Paxton, "The Politics of Mutuality"

2018, 43(2), Hayes, "Touch \& Play: A bit of history and perspective"

2019, 44(1), Beaulieux, "How the First Rule Brougth \#MeToo Contact Improvisation"

2019, 44(2), Beaulieux, (CQ Contact Improvisation Newsletter), "Starting by believing Maria: Responding to Sexual Violence in Safer Brave Contact Improvisation Spaces"

2019, 44 (2), Aramo \& Skrzypczak, "Queer Contact Improvisation (QCI): Alliance and Disruption"

Fuente: Elaboración propia. 
Abordar o no el tema de género -junto al cual o ante el cual, se sitúa el de la sexualidad- ha constituido en sí un dilema preliminar para organizadores y facilitadores de eventos; ya en el congreso europeo de profesores de Contact Improvisation celebrado en Ámsterdam en 1995 identificamos una de las principales paradojas: en lo que resultó un tiempo escaso de discusión, se recogía el sentimiento de que estas cuestiones no se habían hecho explícitas en el desarrollo de esta forma de danza, aun existiendo un creciente interés en abordarlas; precisamente, este manifiesto deseo de debate chocaba con el hecho de que, para algunos, una de las principales atracciones del Contact Improvisation había sido su originaria neutralización de género y sexualidad, es decir, la ausencia o la exclusión del tema (Adkins, Heitkamp, Xanitha y Koopmans, 1996).

A este respecto, Nancy Stark Smith (1996, p. 111), al introducir el segundo monográfico sobre sexualidad e identidad hace referencia a las formas espontáneas de debate que han circulado de forma casual en los espacios de improvisación o en las performances pasando por distintos registros artísticos en comunicación con otras esferas del mundo, de donde vienen y a donde regresan. Por su parte, otros espacios de deliberación se afirman en el contexto académico, un contexto que estaba ya presente inicialmente dado que las primeras exploraciones coreográficas se realizaron en campus universitarios contando con los propios estudiantes como bailarines -Bennington College (N. Little, comunicación personal, septiembre 30, 2018) y Oberlin College (Lori B \& Paxton, 1996, p. 83; Nelson \& Stark Smith, 2008 [1979])-; se puede constatar que se trata de un ámbito institucional, el educativo, que no cesa de involucrarse (BrozasPolo, 2017; Cooper, 1993, 2010). Hay que considerar, igualmente, los círculos de debate que ocurren en los propios grupos de práctica -en los talleres, festivales o jams o sesiones de improvisación-, así como en redes sociales como Facebook, entre otras; se puede señalar, por ejemplo, el grupo denominado Contact Improvisation Facilitators Worldwide Networking Discussion como un foro que ha albergado en los últimos meses prolíficas reflexiones y numerosos interrogantes que compartimos profesores de Contact Improvisation; algunas de las discusiones han sido publicadas posteriormente en Contact Quarterly, como ocurre con la definición de CI purista (Bartley, 2018, pp. 41-43) 
y, recientemente, ha servido para la revisión colectiva de las pautas de las jams -guidelines- para resolver dudas didácticas o para seguir cuestionando el sentido del propio debate sobre la sexualidad "Why to speach about sexuality in a CI space?"-.

\section{Igualitarismo versus neutralización: ¿cuerpos sin género?}

Para poder abordar aquí una de las más determinantes y polémicas de las cuestiones en torno a esta danza colectiva, la propuesta de una neutralización corporal del género -¿cuerpos sin género?-, es preciso recordar que el Contact Improvisation asimila muchos de los rasgos que definen la danza posmoderna; en concreto, se puede identificar con una de sus etapas, la de "metáforas y metafísica", tal como lo establece Banes (2002, pp. 45-66) al referirse a las creaciones propias de los años 70. Se produce en dicho contexto una especie de desafección o distanciamiento del que se consideraba hasta entonces el contenido coreográfico -lo expresado- al tiempo que un interés por explorar la materialidad de los cuerpos; este enfoque encuentra en el Contact Improvisation uno de sus máximos exponentes: la fisicalidad como perspectiva que se identifica con la preponderancia de la percepción del instante presente (Louppe, 2011, p. 145). En términos de Suquet (2006), en el Contact Improvisation cristaliza la concepción de cuerpo como laboratorio de la percepción, pero se trata de un laboratorio con espacio para que resuenen las utopías libertarias de la década anterior. A este respecto, nos preguntamos cómo se puede asociar el énfasis en la percepción del movimiento -el propio y el del otro- con la conciencia política o social: ¿es compatible esta determinación en la conciencia física del cuerpo con otras formas de conciencia?, ¿de qué modo se ven implicadas unas en otras? Ann Cooper (2018) trae a colación el término "políticas de la percepción" para insistir en esta perspectiva de género implícita, pero a veces relegada en la práctica del Contact Improvisation, y en la posibilidad de transferencia de la atención perceptiva a otros ámbitos de la atención. Esta relación entre distintas formas de conciencia va a determinar el devenir de distintos discursos aparentemente opuestos. 
A este respecto, un importante cimiento -aún referente en publicaciones actuales de CI- es el trabajo académico de Cynthia Novack (1990), Sharing the Dance: Contact Improvisation and American Culture, concebido como una aproximación etnográfica; en 1988 apareció ya un anticipo en Contact Quarterly (véase tabla 1). En su tesis, Novack se refiere al Contact Improvisation como una "construcción de cuerpo sin género" que posibilita la percepción de la interacción como no sexual. Ante esta definición se desencadena inmediatamente otro interrogante: ies esta una posible estrategia de igualdad?

Contact Improvisation, which posits as its central technique the physical encounter between two bodies considered as weight and mass, usually conveys sensuality. But its construction of the body as not gendered enables perception of interaction as not sexual (Novack, 1990, p. 163).

Es preciso recordar que el período comprendido entre finales de los 60 y principios de los 70 -cuando surge el Contact Improvisationse considera un momento importante para la teoría feminista y para la consolidación del feminismo radical (Scraton, 1995, p. 19). Precisamente, en este sentido, se asocia al Contact Improvisation con un movimiento de reivindicaciones sociales en el que gran parte de las y los artistas que se relacionaron con los prolegómenos de esta técnica eran activistas -ifeministas?-. En términos de Novack (1990, p. 3), el Contact Improvisation forma parte de los experimentos sociales en igualitarismo y comunidad que ocurrieron en los 60/70 en diversas organizaciones políticas y sociales y, al mismo tiempo, era una de las empresas que en danza, teatro, terapia y preparación física trataban de redefinir el ser como cuerpo inteligente y receptivo.

En este marco ¿cuál fue la primera política corporal del Contact Improvisation respecto a la sexualidad? Novack remarca el distanciamiento del coreógrafo fundador Steve Paxton, respecto de cualquier posicionamiento sobre el género o de la implicación sexual en la práctica del Contact Improvisation. Paxton incide en la importancia de neutralizar para garantizar, entre otros aspectos, la concentración y la seguridad de los bailarines. Sin embargo, esta autora recoge algunas dudas derivadas de las manifestaciones de algunos practicantes entrevistados; de hecho, en este texto pionero (Novack, 1990, p. 172) queda abierto un debate que se mantiene vivo en la revista Contact Quarterly en la actualidad: 
- Por una parte, la práctica de Contact Improvisation permite a muchos bailarines experimentar una sensualidad que disfrutan precisamente porque la práctica no es explícitamente sexual.

- Por otra, algunos hombres homosexuales manifiestan que la práctica de Contact Improvisation les permite experiencias de intimidad -no sexuales- tanto con hombres como con mujeres.

- Asimismo, algunas personas indican que los encuentros sexuales pueden ser más frecuentes entre los bailarines de Contact Improvisation que en otras formas de danza y que, aunque a menudo se minimiza la sexualidad, el hecho de bajar las barreras sociales convierte a los practicantes en personas más vulnerables.

- Ya se señalan, al respecto, posibilidades potencialmente perturbadoras -de una práctica basada intensa y extensamente en el contacto- y cómo algunos jóvenes parecen haber encontrado en el Contact Improvisation un contexto para la exploración sexual.

- Otros practicantes, sin embargo, no consideran que el Contact Improvisation pueda ser un vehículo para encuentros sexuales en mayor grado que cualquier otra forma de danza o actividad social.

Como parte de la crítica al que algunos consideran escaso compromiso político del Contact Improvisation recogemos el testimonio de Diane Torr, entrevistada por Paul Langland en el segundo de los monográficos sobre sexualidad e identidad editado por Contact Quarterly en 1996. Torr declara haber sido marxista feminista en la época en la que fue formada directamente por Steve Paxton y Mary Fulkerson, y manifiesta su asombro ante la posición ¿apolítica? de estos dos maestros en sus respectivas prácticas de Contact Improvisation y Release Technique. Curiosamente, a estas dos técnicas y también al Gaga -lenguaje que desde los 90 desarrolla el coreógrafo israelita Naharin- se refiere Bojana Cvejic (2018, p. 26) cuando alude a la inconsciencia política que puede observarse en una variedad de formas coreográficas de "blanca armonía". La misma autora, refiriéndose específicamente al Contact Improvisation, se pregunta si la relación de dos personas improvisando juntas se puede basar solo en "leyes naturales" (gravedad, fricción, soporte...) sin referencia a "leyes positivas" como las convenciones sociales o las performatividades de género (Cvejic, 2019, p. 63). 
La entrevista entre Dian Torr y Paul Langland ilustra la divergencia de puntos de vista desde los que se puede entender la práctica del Contact Improvisation, así como las distintas formas de abordar la política corporal y coreográfica en relación con el género y la sexualidad: mientras Dian Torr lamenta la ausencia de tratamiento de la sexualidad en su experiencia de aprendizaje del Contact Improvisation, Paul Langland se refiere al potencial político y liberador de esta forma de improvisación cuyo contexto feminista, según él, justifica este aparente distanciamiento de las temáticas de género:

D. I would say that the whole issue of sex and sexual feelings, or even sexual expression are subjects which don't seem to be addressed in CI, except by extremely progressive teachers.

P. I remember so clearly in the early ' 70 when I first started dancing and became entranced with Contact, it was a very political time and it was a time when feminism was first bursting in the scene. [...] For people who wanted to be creative and dancers who were very tactile, this was a blessing -a tremendous liberation to be able to have a safe form of touching where you weren't expected to get genital; at the same time there were issues of the equalization of the roles of men and women in Contact, and that was exciting.

D. Well, I first studied CI in 1974 with Steve Paxton at Dartington College of Arts in England, and I have been practicing CI off and on since then. [...] Sexuality, for me, in indeed part of that not something that should be singled out. In a way it's been singled out because it's never been dealt with. It's been denied. [...] I was a Marxist-Feminist, and I couldn't understand these two people who didn't seem at all interested in class analysis. How could they have a philosophy of life? (Torr \& Langland, 1996, p. 113).

Aunque parece que conversan en dos diálogos paralelos, Torr y su entrevistador están en un mismo diálogo con perspectivas muy diferentes sobre la misma práctica. Algo similar sucede en la conversación que mantienen Lori B y Paxton en 1996 publicada en Contact Quarterly, en la cual se manifiesta un equilibrio de opuestos entre la preocupación por lo sexual de Lori B, psicoterapeuta, y la de Paxton, coreógrafo no experto ni particularmente interesado en la sexualidad:

S.P. [...] But my feeling were, and still are, that I am not somebody who feels he understands sex. I presume it's every body's own trip, and that when mature people come into a room to do a touch-based form, they go 
through all of these trips. I'm not sure it's the responsibility of the teachers or the form to... I mean we're not a psychotherapy group...

LB. I think that's a big question, that issue of responsibility. For instance, coming onto a learning environment which is, by definition, hierarchical [...] (Lori \& Paxton, [2008] 1996, p. 83).

Por su parte, en un sentido crítico próximo al de Torr, Dana Prince (2008 [2003]) introdujo en Contact Quarterly una pregunta que pretende situar la cuestión del género a cierta distancia del ámbito particular de la sexualidad: "¿Hay que dejar la identidad en la puerta?" -de la clase de Contact Improvisation-. Desde la perspectiva de esta autora, sería preciso considerar los significados que para cada persona tiene el contacto. Según ella, en Contact Improvisation se está de acuerdo en criticar en un nivel muy básico las formas en las que el género afecta al contacto; y es cierto que la cuestión del género ha sido abordada -en los últimos años- por su relación con la sexualidad y en particular por la preocupación por las interpelaciones sexuales del tacto. No se ha discutido tanto, según Prince (2008, p. 269), sobre las formas en las que la raza o la historia pueden afectar a distintos grupos de hombres o mujeres, ni sobre las formas en las que el tacto es interpretado de distintas formas por bailarines queer o transgénero. A este respecto, ante la insistencia de algunas de las primeras críticas, Paxton (1989) señalaba que no siempre se puede saber lo que cada persona siente o experimenta y que el Contact Improvisation no conlleva necesariamente valores o relaciones de igualdad ya que la improvisación es algo por hacer -por construir entre quienes lo practican, entendemos-. Ann Cooper (2018, p. 36), por su parte, manifiesta haber sentido durante años un cierto rechazo en las clases de Contact Improvisation al tratamiento de la diferencia cultural, incluyendo género y raza, así como falta de respuesta ante las cuestiones de acoso sexual producidas en el amplio marco sociopolítico de los distintos entornos de la práctica. Una de las consecuencias de esta resistencia a abordar la diferencia ha sido, según ella, la exclusión de determinados sectores sociales en la danza.

El tratamiento verbal o la reflexión sobre una idea o un aspecto de la experiencia, que para unas es necesario y urgente, para otras no tiene lugar o no tiene preferencia en el contexto del aprendizaje o 
de la investigación coreográfica de esta forma. En este sentido, Mary Fulkerson (1996, p. 40) considera la sexualidad como una dimensión no central e incluso una dimensión minimizada intencionalmente por algunos profesores con el fin de reconciliar los roles entre hombres y mujeres; este es otro de los argumentos esgrimidos al respecto junto al de la seguridad mencionado por Paxton (1989). La misma autora insiste en que el discurso original del Contact Improvisation es un discurso no verbal que se actualiza en los diálogos de movimiento; argumenta que la experiencia de esta práctica ha desarrollado a posteriori un discurso escrito por los propios practicantes que ha construido la ideología de esta forma de danza. A este respecto, parece desencadenarse cierto debate entre el discurso verbal, oral o escrito, y el discurso corporal que genera la propia práctica de la danza mientras sucede.

En la misma línea argumentativa, Nancy Stark Smith, preguntada por David Koteen, insiste en que la cuestión del género no es primordial en esta práctica, donde la política respecto a la igualdad es mucho más amplia. El Contact Improvisation es un diálogo que descategoriza dualismos jerárquicos entre grande y pequeño, bailarín o no, joven o viejo, amigo o extraño; es decir, no solo importa el dualismo que afecta a las categorías de hombre/mujer o a la sexualidad: "There is a lot that gets paired in the Contact duet. Gender is one of this things, sexuality another [...] Certaintly one's gender and sense of oneself as a man or a woman is present, but it is not necessarily the primary factor" (Koteen \& Stark Smith, 2008, p. 9).

Otro punto de vista es el de Martin Keogh -reconocido pedagogo de Contact Improvisation-, quien se distancia del pensamiento ortodoxo de Paxton, de Stark Smith y de gran parte del grupo pionero; afirma considerarse un ser sexual también cuando baila Contact Improvisation, resultándole imposible amputar su condición sexual:

Steve Paxton said CI is not a sexual dance... (is not a glande game...) This is not true for me. There is a way in which I am always aware of myself as a sexual being. Every breath I take is sexual. There is a rapture I feel when I'm dancing with women and a pride I feel in being a man when I dance with men. I can't amputate that part of myself. [...] Whenever we dance, there is a testing of what is consensual. Will you accept my weigh? Can we go fast? Can we go very very slow? Occasionally I meet someone and we 
consensually bring an erotic or seductive energy to the dance. We move in concentrically and test what is welcome for both of us. There is a safety in the exchange because we are chaperoned by our sense of appropriate behavior in the jam environment (Keogh, 2018, p. 276).

A partir de esta afirmación y de la constatación de experiencias de abuso sexual en el contexto de alguna jam de su propia comunidad en los años ochenta, Keogh desarrolla una propuesta didáctica para aprender a poner límites en la danza y desarrollar el consenso en un diálogo físico que no tiene porqué excluir el componente erótico: 101 formas de decir no en CI. Otras pautas similares -guidelines- habían sido ya planteadas con anterioridad (Elliot, 1990), aunque la de Keogh es de las más mencionadas; al haberse publicado en varios formatos y ediciones -la última en un capítulo de su libro Dancing Deeper Still (Keogh, 2018)- se suele citar como un punto de partida para la elaboración de pautas de actuación en la práctica. La citada propuesta de Keogh ha sido, sin embargo, muy contestada en los últimos números de Contact Quarterly. Yardley (2017), bailarina de Canadá, ha publicado un documento con experiencias de mujeres afectadas en contextos de Contact Improvisation donde propone un amplio listado de sugerencias de actuación y en el mismo año surgieron otras publicaciones similares (Gottlieb, 2017). Beaulieux, por su parte, desde Estados Unidos rebate detalladamente aspectos como el principio de autorresponsabilidad formulado por Keogh, conocido como "la primera regla"; para Beaulieux (2019a) no solo no es una norma suficiente, sino que puede ser perniciosa pues sitúa en segundo lugar aspectos primordiales como la responsabilidad de todo el grupo, la de los organizadores y, en particular, la de las personas irrespetuosas en la danza. Apoyando una interrupción-protesta de la jam de Berkeley en 2018, insiste en reclamar atención sobre un tema conflictivo que hasta ahora había sido considerado anecdótico o relativo a contextos muy determinados (Beaulieux 2019a, 2019b); con estos artículos exige la revisión de la cultura de la jam en cada comunidad y, ciertamente, el debate junto a otras acciones colectivas se están desencadenando, lo que queda de manifiesto en varios blogs, como los relacionados con el movimiento $\#$ metoo $^{2}$ o con la cultura

2. https://dancersgroup.org/2018/06/contact-improvisers-consider-metoo/ 
del consenso. ${ }^{3}$ La propia revista CQ (2014) ya había propuesto años antes un espacio para compartir las orientaciones para las jams de cada grupo organizador, que no deja de actualizarse.

\section{Feminismo y masculinidades en Contact Improvisation}

Son diversas las relaciones que con el feminismo se establecen en el marco teórico-práctico del Contact Improvisation desde la perspectiva de los hombres. En buena medida, al disolver los roles de los portés de danza clásica -donde el bailarín ocupa el espacio central y carga con el peso de la bailarina-, o de los bailes de pareja -donde el hombre invita y guía el movimiento-, esta práctica contribuye a evidenciar la construcción social de la masculinidad y a cuestionar los patrones de género imperantes. Por otra parte, en consonancia con la reivindicación "feminista" de Fulkerson (1996) señalada anteriormente, en el Contact Improvisation se desarrolla un discurso de hombres que analiza la identidad masculina y responde al movimiento feminista de los años 60 y 70 desde su propia subjetividad.

En este sentido, resultan esclarecedoras las reflexiones de Burt (2006) a propósito de la masculinidad en los solos coreografiados por Steve Paxton. De Flat (1964), una de las primeras creaciones, Burt (2006, p. 131) advierte de la revelación de un cuerpo masculino desmantelado de los efectos eróticos debido a la ausencia de desarrollo o clímax y donde predomina también la ausencia de tensión física a través de movimientos estrictamente cotidianos. En los solos siguientes -Goldberg Variations (1986), English Suite (1992)- se mantendría el rechazo a las expectativas del juego masculino; según este autor, Steve Paxton profundiza en una propuesta escénica sin jerarquías iniciada por Merce Cunningham: "Paxton desarrolla el uso de formas no jerárquicas de estructuración del grupo y uno de sus propósitos era el de hacer coreografías donde las diferencias sexuales no fueran importantes o significativas" (Burt, 2006, p. 133; mi traducción).

3. https://contactimprovconsentculture.com/ 
Steve Paxton estuvo en la compañía de Cunningham entre 1961 y 1964, pero además en la Judson Church, entre 1962 y 1964; parece ser sobre todo en el seno de este último grupo donde se planteó más explícitamente el intento de crear situaciones de danza que fueran relativamente no jerárquicas (Novack, 1990, p. 207). Las políticas de igualdad de la Judson Church también las aborda Foster (2001) en relación con el Contact Improvisation, las cuales serán recogidas más adelante por otros autores como Curtis (2015). El propio Paxton (2008 [1994], p. 27) explica, además, en Contact Quarterly su presencia en el colectivo The Grand Union (1970-76), un grupo creado inicialmente por la artista Yvonne Rainer que proponía la disolución del liderazgo en un intento fugaz de creación colectiva y democrática.

En las primeras investigaciones que Steve Paxton desarrolló en el Oberlin College en enero de 1972 participó exclusivamente un grupo de hombres (Nelson \& Stark Smith, 2008); pero en las primeras presentaciones en la John Webery Gallery de Nueva York en julio del mismo año, cuando se utilizó por primera vez el término de Contact Improvisation, ya se habían incorporado mujeres como Nancy Stark Smith o Barbara Dilley (Burt, 2006, p. 133). Al parecer, el grupo The Grand Union, formado por algunos y algunas de las artistas que estuvieron años antes en la Judson Church, estaba haciendo una residencia en el Oberlin College y las coreógrafas de la compañía decidieron organizar un curso solo para mujeres. Según Burt, esta circunstancia hizo que Paxton se encontrara a sí mismo trabajando con un grupo de ocho hombres en el gimnasio a los que enseñó una estructura de improvisación que sería la matriz de Magnesium (1972), la primera performance de Contact Improvisation. Otras versiones (P. Peñuela, comunicación personal, junio 5, 2019) indican que Paxton decidió expresamente seleccionar a un grupo de bailarines lo bastante atléticos y entrenados para dicha pieza en la que pretendía explorar los impactos directos de los cuerpos en movimiento: ¿excluyó deliberada o fortuitamente a las mujeres? En efecto, en el video de Cvejic y Laberenz (2013), expuesto en la exposición Steve Paxton: Drafting interior Techinques en el centro Azkuna de Bilbao a la que pudimos asistir en febrero de 2020, Paxton declara que "con las chicas no hubiera hecho un experimento tan rudo". 
En una entrevista con Lori B, Paxton explica el proceso de su trabajo previo en solo, el encuentro en Oberlin en enero de 1972 con el grupo de hombres jóvenes, así como los eventos de Bennington en primavera y Nueva York en junio del mismo año con grupos mixtos. Paxton aclara en este texto la evolución inicial de su propuesta hacia el movimiento colectivo a través de la cooperación en contacto -un contacto que pasa de ser breve e intenso a más continuo, lento y sensible, pero sin atención a los aspectos eróticos-:

SP. I had been working for two years on a solo, which was to free up my movement and get used to what I now call the "sphere of movement" [...] And I tried to teach it to some men at Oberlin College in a class. This work became the performance called Magnesium [...].

LB. Were you working with slow contact at that point?

SP. No. Magnesium happened in January'72. I began working much slowly that spring at Bennington College. The next performance event happened in June with students from Bennington, Rochester, Oberlin, and dancers from New York.

LB. Mixed gender?

SP. Yeah, mixed gender. And one love affair developed in that group. I think I started off from the erotic quality of their touch. I got to watch them working in this form which is so much about cooperation and touch and communication and a kind of almost extra-sensory awareness of other people (Lori \& Paxton, 2008, p. 83).

A propósito de la masculinidad en Magnesium, Curtis (2015, p. 131) considera que la combinación de fuerza, salvajismo, riesgo físico, contacto íntimo no sexual, sensibilidad propioceptiva y colaboración física basada en la confianza convirtieron al Contact Improvisation en un foro ideal para desestabilizar los roles tradicionales de contacto. Según Miller (cfr. Curtis, 2015) en el siglo XXI puede ser más urgente que en el siglo xx construir espacios de enseñanza en contextos específicos de género con el fin de ofrecer a los hombres alternativas a las narrativas y a los comportamientos heteronormativos.

Curtis (2015, p. 130) define el Movimiento de hombres que se generó en Estados Unidos entre los setenta y los noventa como una respuesta o extensión respecto de los movimientos feministas de los 
sesenta y los setenta. Este movimiento se conformó con un amplio rango de actividades y actores que incluían cientos de grupos de amplio espectro sociopolítico, tanto progresistas como conservadores. Curtis constata una interacción entre algunos de estos grupos y las prácticas de Contact Improvisation. En este sentido, se apoya en la tesis de Foster (2001) para afirmar que el Contact Improvisation tiene un especial significado en términos de exploración y deconstrucción de la aceptada corporeización o incorporación de roles de género.

Desde esta perspectiva, en la que también se cruzan sensibilidades gay y queer, se ha considerado al Contact Improvisation como una práctica corporal de gran potencial, junto con otras técnicas y aproximaciones vivenciales o artísticas. Se convierte en herramienta o referencia de experimentación para algunos grupos de activistas que propugnan nuevas políticas corporales así como otras políticas de género y sexualidad: "In the last two years, I've made a number of experiments bringing together information and experience from the Contact Improvi/Body-based Art world and the SexPositive/Body Activist world" (Curtis, 1996, p. 93). Cercano al de Curtis, se encuentra el activismo de Keith Henessy, quien siendo miembro del mismo colectivo artístico en los noventa (Contraband) ha participado en el discurso crítico de género a lo largo de los años en la revista Contact Quarterly (véase tabla 1) y ha mantenido un diálogo crítico permanente con Nancy Stark Smith por diferentes medios; también por medio del audiovisual (Henessy, 2012), donde plantea una serie de preguntas que se han materializado en una investigación específica sobre el cuestionamiento político de esta forma de danza (Hennessy, 2018).

Junto a este discurso de hombres se encuentran otros muy distintos, como el de las mujeres que consideran el Contact Improvisation un modelo, un referente de negociación envidiado, en ese sentido, por las practicantes de otros bailes de pareja como el tango (Brickhill, 2016). También se considera un punto de inflexión en la historia de las relaciones de confrontación sexual entre hombres y mujeres en la danza (Assis y Saraiva, 2013); tendría, en este sentido, el potencial de desnaturalizar suposiciones e imágenes de género y redefinir posibilidades de acción tanto para ellos como para ellas. 


\section{La insistencia queer: ¿no era ya el $\mathrm{Cl}$ una danza queer? o ¿es heteronormativa?}

El discurso queer, que se asocia tanto al movimiento feminista como a algunas reivindicaciones de grupos de hombres o del movimiento LGTBIQ, puede entenderse como una forma de superación de dualidades, en la medida en que rompe con los debates representativos de las posiciones de las mujeres frente a las de los hombres. Se trata de una acepción que, según Butler (2002, p. 315) ha sufrido una reapropiación y una inversión positiva, con la posibilidad de pasar de ser un término despectivo a convertirse en un término emblemático de reparación histórica. Bajo nuestro punto de vista, la disolución de la heteronormatividad que propone la teoría queer se podría llegar a identificar -con cierta simplificación- con la neutralización de la propuesta inicial del Contact Improvisation.

Como señala Horrigan (2017, p. 39) en Contact Quarterly, se produce una interesante tensión en torno al género en Contact Improvisation. Por una parte, el Contact Improvisation parece no tener nada que ver con el género, al no existir roles asignados; pero, por otra, al llevar toda nuestra "humanidad" en la danza cuando improvisamos, también incluimos nuestra identidad de género. El género, en este sentido, configura nuestra organización corporal y espacial. Por ello, es posible observar las dinámicas heteronormativas de género en muchas de las jams de Contact Improvisation: los patrones de movimiento se despliegan mientras bailamos, a veces en contradicción con nuestras propias intenciones como improvisadores. El género afecta en gran medida a nuestra organización e imaginación corporal, a nuestro pensamiento sobre nuestras habilidades o a los roles que desarrollamos en las relaciones. En este discurso de género, Horrigan (2017) se apoya a su vez en Iris Marion Young (2005), quien describe detalladamente los patrones de espacialidad y movilidad de las mujeres como opuestos a los masculinos. De estos patrones no parece fácil desprenderse bailando Contact Improvisation: aunque esta estructura nos impulse a la superación de dicha dualidad de género nuestra educación y nuestra cultura nos recompensa cuando nos conformamos con el modelo dominante de género, por lo que cuesta resistir o no asumir dicho modelo. 
Como ha sido mencionado en apartados anteriores, el Contact Improvisation como técnica de danza o como coreografía abierta, nos reta a incorporar un diverso rango de cualidades y habilidades que atraviesan todo el espectro de género: recibir y dar peso, iniciar y seguir, usar la fuerza y la dulzura, sentir y actuar, etcétera. En este sentido, según Horrigan (2017, p. 42), la técnica de Contact Improvisation activa el género queer, al combinar las cualidades codificadas como masculinas y femeninas, invitándonos a ir más allá del binario; en este sentido se podría hablar del Contact Improvisation como una forma de danza queer en relación con identidades y orientaciones que desbordan el paradigma heteronormativo.

Sin embargo, no son pocas las afirmaciones y experiencias que se refieren al Contact Improvisation como una práctica que se desarrolla en escenas predominantemente heteronormativas y esta puede ser la razón por la que podemos hoy en día encontrar espacios de práctica y de debate específicamente queer, que se autodenominan como tales:

Contact Improvisation is a dance form that prioritizes touch, weight and the bodily listening as guiding principles for dancing together. No leader no follower, the decisions are taken in the moment of the meeting. Some people call it an art-sport. C-I was proposed by Steve Paxton in 1972 and was developed by men and women around him. Since then the practice grows in a mainly heteronormative scene (Village Berlin \& Stretch Festival, 2016).

En el marco de una teoría queer de la danza, Doran George (2017, p. 90) afirma que el Contact Improvisation se distancia del feminismo de los años 70 en el que aparentemente se inscribe, al desarrollar una propuesta de minimización o desgenerificación en lugar de enfatizar la diferencia de género en la construcción del mismo. Según esta autora, el énfasis en las posibilidades mecánicas y en la ejecución cinética propone una actuación escénica de la autenticidad corporal o personal que parece desplazar relaciones de poder culturalmente impuestas; pero, por lo que estamos constatando, en la evolución y desarrollo de la práctica del Contact Improvisation, dichas relaciones de poder, previamente establecidas y enraizadas en cada grupo social, parece que no se dejan desplazar tan fácilmente.

Por su parte, Olaya Aramo y Wiktor Skrzpczak (2019, p. 50) consideran que una de las aportaciones más significativas de las 
prácticas queer al Contact Improvisation es la construcción de discurso y, en particular, de discurso creado colectivamente. Acuñan el término Queer Contact Improvisation para referirse a un campo específico de intersección entre las prácticas queer y el CI, entendiendo que este aporta, junto a otras prácticas somáticas, todo un conocimiento sobre el cuerpo y las dinámicas de la interacción corporal. Este campo se caracterizaría, además, según Papineau y Engelman (2019), por reconocer las fuerzas sociopolíticas como parte de la física.

\section{Persistencia del debate, resistencias y divergencias: ¿el pensamiento o la danza?}

Han pasado cerca de 50 años desde que comenzaran las dinámicas coreográficas promotoras del Contact Improvisation y el pensamiento sigue evolucionando en una práctica que se extiende y aún parece que quiere continuar democratizándose. La popularización -ligada a contextos educativos o recreativos más que estrictamente artísticos (Brozas, 2016, 2017)- conlleva con frecuencia la aparición de inusitados problemas -algunos de los cuales nos atrevemos a asociar con la masificación de la práctica- y, en cualquier caso, el surgimiento de nuevos cuestionamientos para incomodidad, en ocasiones, de las élites de esta danza. El tamaño de los colectivos que investigan o bailan, como un factor condicionante o distorsionante, ya había sido señalado por Paxton (1989, p. 167) en una de sus respuestas a las demandas de explicación o petición de cuentas ante los incidentes de abusos y la percepción de desigualdades en la práctica.

La técnica de Contact Improvisation es explícitamente igualitaria en su conformación coreográfica, en las reglas del juego físico que plantea, pero no otorga una vacunación universal que inmunice a quien baila frente a los juegos de poder inherentes a la cultura del grupo humano en el que se practica. En algunos grupos de Contact Improvisation se puede observar un cierto discurso autocomplaciente, una cierta tendencia a pensar que esta forma (nos) exime de cualquier posibilidad de abuso, conflicto, interés económico, etcétera. Ocurre así, en ocasiones, que la cuestión de la desigualdad de género se tiende a dar por solucionada, como si fuera una danza en la que no cabe dicho debate: así se manifestó alguno de los asistentes del Encuentro 
de Maestros y Organizadores de Contact Improvisation (EMOCIE) celebrado en Navarra en octubre de 2018, que prefirió no participar en el debate de género que se desarrolló por una gran mayoría interesada: ¿puede mantenerse el Contact Improvisation como una isla exenta de reivindicaciones feministas?, ¿es aún necesario que se encuentre presente este -para algunos- cansino lamento de la desigualdad?

El discurso feminista va configurándose lenta pero decididamente, abriendo distintas capas de esta forma de danza: mientras en los años $70 \mathrm{u} 80$ el hecho de bailar sin roles establecidos ni normas restrictivas dirigidas a los practicantes de distinto sexo o género era ya un logro -y lo sigue siendo hoy si se compara con otras formas de danza más ancladas en tradiciones machistas-, en el siglo XXI, afortunadamente, existe una mayor conciencia social y una mayor sensibilidad; una sensibilidad que exige, sobre todo, cuestionarse cómo seguimos afectados por los discursos hegemónicos heterosexuales y cómo estos siguen condicionando las relaciones entre los cuerpos en los marcos de la improvisación coreográfica.

Pensamos por ello en la paradoja de la potencial fragilidad del Contact Improvisation debido a su permeabilidad cultural: un paradigma de cambio social parece definido en su esencia coreográfica, pero cada cuerpo y cada colectivo que baila/discute alberga una historia y una identidad cultural. Sería la conciencia de esta heterogeneidad la que nos permitiría la observación de las distintas dinámicas culturales en las que se efectúa o se materializa la danza.

A día de hoy para una -ipequeña?- parte de los practicantes de Contact Improvisation, tanto el debate del género como el de la sexualidad resultan fuera de lugar en los círculos de discusión que acompañan la práctica, mientras que para otra parte -iimportante?de la comunidad bailarina y en particular de quienes nos dedicamos a enseñar y organizar, el diálogo resulta ineludible, en línea con la evolución de los debates en Contact Quarterly. Aquí la cuestión sería acordar cómo y dónde ha de tener lugar el debate. Resulta llamativa la publicación de dos textos del mismo año y la misma autora -Karen Nelson- aparentemente antagónicos: en el primero solicita espacio en la sección Still Moving de la revista Contact Quarterly para abordar el tema de las apropiadas o inapropiadas conductas sexuales que pueden interferir en la danza (Nelson, 1994a) y en el segundo 
solicita que se respete el espacio de danza solo para bailar y que se desarrollen los debates verbales en otro lugar (Nelson, 1994b):

Perhaps "Still Moving" in CQ could provide an arena for a discussion of issues regarding appropriate and inappropriate sexual behavior as it interfaces with contact dancing (Nelson, 2008 [1994a], p. 30).

We invite people to come to practice the discipline of dance and, while people bring their own personal opinions through which they view the world (real or otherwise), we agree to agree that we are here to dance. We should also agree that we can potentially disagree about everything else, and act with that in mind. Hopefully, each person realizes the intent and extent of the situation and willingly engages themselves (Nelson, 2008 [1994b], p. 38).

Algunos bailarines (K. Frost, comunicación personal, mayo 18, 2018), por su parte, han manifestado sentir en el contexto del Contact Improvisation, incluso desde la propia revista CQ, cierta resistencia a introducir una reflexión sobre género/sexualidad que en estos momentos es ya irrefrenable y cuestiona la propia práctica del Contact Improvisation, una práctica que se viene considerando a su vez paradigmática en la igualdad, alternancia o desaparición de roles de géneros ligados en su técnica a dirigir/seguir, sostener/ser sostenido.

Según Paxton, "la libertad y la igualdad estaban presentes en el momento de la creación del Contact Improvisation, lo que ocurre al momento siguiente [en el devenir de estas décadas], es algo que debemos -y especialmente los antropólogos deben- ponderar" (1989, p. 167; mi traducción). Más recientemente, en una discusión sobre el significado del "CI purista" Paxton invita, a quien tenga otras propuestas distintas a lo que ellos definieron con el término Contact Improvisation, a utilizar otro nombre:

We started practicing in ' 72 . We invented the form and the name. The name did not exist before that time, and since that time we have been charged or define it and given the responsibility to tell our truth about that invention. I amazed to be confronted with "CI Purist", and seek to discover the perspective from which that phrase is launched. Who seeks to redefine or qualify CI? Why do they offer that modifies the intent and long-term practice of the form? And why don't they just invent their own name for whatever it is they propose? (Bartley, 2018, p. 43). 
Y ciertamente, están surgiendo otros términos que lo matizan, complementan o precisan como Early CI \& Post CI (Hennessy, 2012, 2018), así como Radical Contact o Queer Contact. Otra acepción más sería la de Touch and Play que, aunque no es exactamente Contact Improvisation, no podemos dudar de que está en sus intersecciones (Hayes, 2018) o en sus derivaciones.

En ocasiones nos identificamos con quienes prefieren continuar la danza y relegar la palabra -como Nelson (1994b)-, pero también con quienes perciben la urgencia de la irrupción verbal apoyando a las mujeres afectadas -como Beaulieux (2019a, 2019b)-. En general, en cuanto que enseñantes -como Curtis (1996)- creemos que es nuestra obligación abordar el tema. Tanto la danza como el debate en el caso del Contact Improvisation parecen imparables e inseparables: danza y pensamiento, acción colectiva y deliberación, van encontrando espacios comunes de afirmación. La diversidad de los discursos abordados, en la medida en que estos afirman o cuestionan la igualdad de género desde posiciones aparentemente antagónicas, también contribuye a la democratización de la danza $\mathbf{I}$

\section{Referencias}

Adkins, J., Heitkamp, D., Xanitha, Z. \& Koopmans, W. (2008 [1996]). Study Lab on CI and Sexuality at the $10^{\text {th }}$ European Contact Teachers Conference. En N. Stark Smith y L. Nelson (Eds.), Contact Quarterly's Contact Improvisation Sourcebook II: Collected Writings and Graphics from Contact Quarterly Dance Journal 1993-2007 (pp. 101102). Contact Editions.

Aramo, O. \& Skrzypczak, W. (2019). Queer Contact Improvisation (QCI): Alliance and Disruption. Experiences and reflections from the QCI Symposium and Festival Hamburg 2018. Contact Quarterly, 44(2), 5053. https://contactquarterly.com/cq/article-gallery/view/queer-contactimprovisation-qci-alliance-and-disruption.pdf

Assis, M. P. y Saraiva, M. C. (2013). O feminine e o masculine: das origens do balé à contemporaneidade. Movimento, 19(2), 303-323. https:/doi. org/10.22456/1982-8918.29077

Banes, S. (2002). Terpsichore en baskets. Post-modern dance. Chiron. 
Bartley, C. (2018). What is a CI "Purist"? Contact Quarterly, 43(2), 41-43. https://contactquarterly.com/contact-improvisation/newsletter/view/ what-is-a-ci-purist $\# \$$

Beaulieux, M. (2019a). How the First Rule Brought \#Me Too to Contact Improvisation. Contact Quarterly, 44(1), 46-50. https://contactquarterly. $\mathrm{com} / \mathrm{cq} /$ article-gallery/view/how-the-first-rule-brought-metoo-tocontact-improvisation.pdf

Beaulieux, M. (2019b). Starting by Believing Maria: Responding to Sexual Violence in Safer Brave Contact Improvisation Spaces. CQ Contact Improvisation Newsletter, 44(2) https://contactquarterly.com/contactimprovisation/newsletter/view/starting-by-believing-maria\#\$

Brickhill, E. (2016). Argentine Tango and Contact Improvisation. [Tesis de Maestría, Universidad de Wollongong]. http://ro.uow.edu.au/ theses/4728

Brozas, M. P. (2000). Contact Improvisation: danza, acrobacia y pedagogía corporal. En J. P. Fuentes y M. Macías (Eds.), Actas I Congreso de la Asociación Española de Ciencias del Deporte (pp. 309-316). Universidad de Extremadura.

Brozas-Polo, M. P. (2016). Expansión geográfica y conceptual de la danza Contact Improvisation en España (1990-2000). Retos, (30), 30-35. https://bit.ly/2ZEF2go

Brozas, M. P. (2017). Los espacios como dispositivo de la construcción de prácticas corporales y coreográficas contemporáneas. A propósito del contact improvisation en el contexto español. Revista de Dialectología y Tradiciones Populares, 72(2), 397-421. https://doi.org/10.3989/ rdtp.2017.02.005.

Burt, R. (2006). Steve Paxton and Contact Improvisation. En The Male Dancer. Bodies, Spectacle, Sexualites (pp. 130-135). Routdledge.

Butler, J. (2002). Cuerpos que importan. Paidós.

Cooper Albright, A. (1993). Contact in Institutions. Contact Quarterly, 18(1), 70-72.

Cooper Albright, A. (2010). Encounters with Contact. Dancing Contact Improvisation in College. Oberlin College Theater and Dance Program. 
Cooper Albright, A. (2018). Politics of Perception. En CI Intersections. AQuestion from CQ and a Round of Responses. Contact Quarterly, 43(2), 35-36.

CQ Eds. (2014). Jam Guidelines. CQ Contact Improvisation Newsletter, 39(2). https://contactquarterly.com/contact-improvisation/newsletter/ view/jam-guidelines $\#$

Curtis, J. (1996). Why Talk (Dance, Painting) about Sex? Contact Quarterly, 21(1), 53-57.

Curtis, J. (2015). Movement in the Men's movement: Contact Improvisation and social change. Journal of Dance $\mathcal{E}$ Somatic Practices, 7(2), 129-142. https://doi.org/101386/jdsp.7.1.129_1

Cvejic, B. (2018). Aesthetic Obfuscations in White Western Dance. En Dance Studies Association Conference "Contra: Dance \& Conflict" (p. 26). Dance Studies Association.

Cvejic, B. (2019). A physical quest for natural rights. En R. Bigé (Ed.), Steve Paxton: Drafting Interior Techniques (pp. 52-66). Culturgest.

Cvejic, B. \& Laberenz, L. (2013). ... In a non-wimpy way... [archivo de video]. Musée de la Danse. http://www.bojanacvejic.info/in-a-nonwimpy-way/

Elliot, D. (1990). Making Safe Contact. Contact Quarterly, 15(2), 41-44.

Foster, S. (1998). Choreographies of Gender. Signs, 24(1), 1-33.

Foster, S. (2001). Improviser l'autre: spontanéité et structure dans la danse experimentale contemporaine. En M. Febvre y I. Ginot (Eds.), Danse et altérité (pp. 25-37). Protée.

Fulkerson, M. (1996). Taking the Glove Without the Hand: An Historical Perspective. Contact Quarterly, 21(1), 40-42.

George, D. (2017). The Hysterical Spectator. Dancing with Feminists, Nellies, Andro-Dykes, and Drag Queens. En Queer Dance: MeaningEMakings (pp. 83-108). Oxford University Press.

Gottlieb, S. (2017). Myths to Break Down Moving Toward Ethical Communication and Ethical Sexuality in CI. Contact Improvisation Blog. https://bit.ly/3eK3CAu

Hayes, D. (2018). Touch \& Play: A Bit of History and Perspective. Contact Quarterly, 43(2), 40. 
Hechler, A. (2008). Heteronormativity in Contact Improvisation. Contact Quarterly Unbound, (33). https://contactquarterly.com/cq/ unbound/\#year=2008

Hennessy, K. (2012). Keith Hennessy talks with Nancy Stark Smith nov 12 [archivo de video]. YouTube. https://www.youtube.com/ watch?v=KOCtxbpErYs

Hennessy, K. (2018). Questioning Contact Improvisation. Circo Zero.

Horrigan, K. (2017). Queering Contact Improvisation. Addressing Gender in CI Practice and Community. Contact Quarterly, 42(1), 39-43.

Keogh, M. (2008 [2003]). 101 Way to Say No to Contact Improvisation: Boundaries and Trust. En Contact Improvisation Sourcebook. Collected Writing and Graphics from Contact Quarterly Dance Journal 1975-1992 (pp. 273-276). Contact Editions.

Keogh, M. (2018). The practice of Contact Improvisation. Dancing Deeper Still. Intimately Rooted Books.

Koteen, D. \& Stark Smith, N. (2008). CaughtEFFalling. The Confluence of Contact Improvisation, Nancy Stark Smith, and Other Moving Ideas. Contact Quarterly Editions.

Lori, B. y Paxton, S. (2008 [1996]). The sex issue. Lori B \& Steve Paxton interview each other. En Contact Improvisation Sourcebook: Collected Writing and Graphics from Contact Quarterly Dance Journal 1975-1992 (pp. 81-89). Contact Editions.

Louppe, L. (2011). Poética de la danza contemporánea. Ediciones Universidad de Salamanca.

Nelson, K. (2008 [1994a]). Dear CQ. Arena for discussion. En Contact Improvisation Sourcebook: Collected Writing and Graphics from Contact Quarterly Dance Journal 1975-1992 (p. 30). Contact Editions.

Nelson, K. (2008 [1994b]). Jam Organizer's Sanity Clause. En Contact Improvisation Sourcebook: Collected Writing and Graphics from Contact Quarterly Dance Journal 1975-1992 (p. 38). Contact Editions.

Nelson, K. (2008 [1996]). Touch Revolution: Giving Dance. En N. Stark Smith y L. Nelson (Eds.), Contact Quarterly's Contact Improvisation Sourcebook II: Collected Writings and Graphics from Contact Quarterly Dance Journal 1993-2007 (pp. 103-105). Contact Editions. 
Nelson, L. y Stark Smith, N. (2008 [1979]). A Short History. En Contact Improvisation Sourcebook: Collected Writing and Graphics from Contact Quarterly Dance Journal 1975-1992 (p. 2). Contact Editions.

Novack, C. J. (1990). Sharing the Dance: Contact Improvisation and American Culture. University of Wisconsin Press.

Papineau, E. y Engelman, S. (2019). Creating Space for Queer Contact Improvisation. CQ Contact Improvisation Newsletter, 44(1). https://bit. ly/2WvzUc8

Paxton, S. (1975). Contact Improvisation. The Drama Review, 19(1), 40-42.

Paxton, S. (1989). Hier-Visibility. Contact Quarterly, 14(1), 166-167.

Paxton, S. (2008 [1994]). Two Book Reviews. En N. Stark Smith y L. Nelson (Eds.), Contact Quarterly's Contact Improvisation Sourcebook II: Collected Writing and Graphics from Contact Quarterly Dance Journal 1993-2007 (pp. 27-29). Contact Editions.

Prince, D. (2008 [2003]). Leave Your Identity at the Door? Politics of Difference and Contact Improvisation. En N. Stark Smith y L. Nelson (Eds.), Contact Quarterly's Contact Improvisation Sourcebook II: Collected Writing and Graphics from Contact Quarterly Dance Journal 1993-2007 (pp. 268-269). Contact Editions.

Rizzo, F. (1998). Con el sudor de tu frente. Tiempo de danza, (16), 35-40.

Scraton, S. (1995). Educación física de las niñas: un enfoque feminista. Morata.

Stark Smith, N. (2008). Preface. En N. Stark Smith y L. Nelson (Eds.), Contact Quarterly's Contact Improvisation Sourcebook II: Collected Writings and Graphics from Contact Quarterly Dance Journal 1993-2007 (p. IX). Contact Editions.

Stark Smith, N. (2008 [1996]). Who do we think we are? En N. Stark Smith y L. Nelson (Eds.), Contact Quarterly's Contact Improvisation Sourcebook II: Collected Writings and Graphics from Contact Quarterly Dance Journal 1993-2007 (p. 111). Contact Editions.

Stark Smith, N. (2008 [1998]). A Question of Copyright -Some history-. En N. Stark Smith y L. Nelson (Eds.), Contact Quarterly's Contact Improvisation Sourcebook II: Collected Writings and Graphics from Contact Quarterly Dance Journal 1993-2007 (p. 177). Contact Editions. 
Suquet, A. (2006). Escenas. El cuerpo danzante: un laboratorio de la percepción. En J. J. Courtine (Ed.), Historia del cuerpo: Las mutaciones de la mirada. El siglo xx (pp. 394-397). Taurus.

Torr, D. \& Langland, P. (2008 [1996]). Between the Cracks: Gender \& Sexuality in the New Dance/Performance Scene. En N. Stark Smith y L. Nelson (Eds.), Contact Quarterly's Contact Improvisation Sourcebook II: Collected Writings and Graphics from Contact Quarterly Dance Journal 1993-2007 (pp. 112-121). Contact Editions.

Village Berlin \& Stretch Festival (2016, diciembre 26). Queer Contact Improv Laboratory. Facebook. https://www.facebook.com/ events/1526115000735971/

Yardley, B. (2017). Respecting Boundaries/Coexisting Genders: A Zine about Women's Experiences of Feeling Unsafe in Contact Improv. CQ Contact Improvisation Newsletter 42(2). https://bit.ly/32xh9ZG

Young, I. M. (2005). On Female Body Experience: Throwing Like a Girl and other Essays (Studies in Feminist Philosophy). Oxford University Press. 